Research Article

\title{
Electrocardiography Interpretation Competency of Medical Interns: Experience from Two Ethiopian Medical Schools
}

\author{
Melaku Getachew (D), ${ }^{1}$ Temesgen Beyene, ${ }^{2}$ and Sofia Kebede ${ }^{2}$ \\ ${ }^{1}$ Department of Emergency Medicine and Critical Care, Haramaya University, Harar, Ethiopia \\ ${ }^{2}$ Department of Emergency Medicine and Critical Care, Addis Ababa University, Addis Ababa, Ethiopia
}

Correspondence should be addressed to Melaku Getachew; melakug02@gmail.com

Received 27 February 2020; Revised 10 April 2020; Accepted 30 April 2020; Published 11 May 2020

Academic Editor: Mario Ganau

Copyright ( $) 2020$ Melaku Getachew et al. This is an open access article distributed under the Creative Commons Attribution License, which permits unrestricted use, distribution, and reproduction in any medium, provided the original work is properly cited.

\begin{abstract}
Background. Electrocardiography (ECG) is the graphical display of electrical potential differences of an electric field originating in the heart. Interpretation of ECG is a core clinical skill in the department of emergency medicine. The main aim of this survey was to assess competency of ECG interpretation among 2018 graduating class medical students in Addis Ababa University and Haramaya University. Methodology. A cross-sectional survey was conducted on medical interns at Addis Ababa University and Haramaya University. Data had been collected from October 01, 2018, to October 30, 2018, by using structured questionnaires. Data were entered, cleaned, edited, and analyzed by using SPSS version 25.0 statistical software. Descriptive statistics, cross-tabs, chi-squared test, Mann-Whitney $U$ test, and binary logistic regression were utilized. Results. Two-hundred and two graduating medical students were involved on this survey, out of which 61.3\% (95\% CI 56.3-66.3\%) and 32.75\% (95\% CI 28.25-37.25) were able to correctly interpret the primary ECG parameters and the arrest rhythm of ECG abnormalities, respectively. The ability to detect from common emergency ECG abnormalities of anterioseptal ST segment elevation myocardial infraction, atrial fibrillation, and first-degree atrioventricular block was $42.6 \%, 39.1 \%$, and $32.1 \%$, respectively. Conclusion. This survey showed graduating medical students had low competency in ECG interpretations.
\end{abstract}

\section{Introduction}

Electrocardiography (ECG) is the graphical display of electrical potential differences of an electric field starting from the heart. It is a frequently used investigation for the diagnosis of heart disease and electrolyte abnormalities. Most of the patients who visit the emergency department (ED) had ECG abnormalities. Accurate interpretation of ECG is an essential clinical skill in emergency and critical care medicine [1-3]. In day to day clinical practice, it is important for physicians and graduating medical students to know the accuracy of their ECG interpretation skills to correctly rule out the presence of cardiac disease or manage their patients $[4,5]$. Even though there was no research done in Ethiopia or Africa regarding ECG interpretation skill of graduating medical students, around 9\% of death in Ethiopia was caused by cardiovascular disease [6]. In Portugal, where undergraduate programs are firmly established, the overall accuracy of general practitioners (GP) for detecting ECG abnormalities were $81.0 \%$ [7]. The overall missed case rate for all seven ED was $12.8 \%[8,9]$.

ECG interpretation skills vary among medical students, GP, and specialists. Accurate diagnosis of ECG abnormalities by a physician in any specialty contributes to appropriate clinical decision-making. Correct ECG interpretation assumes technical standards adhered to during the acquisition and recording of tracings. Many technical and patientrelated factors may alter the quality of recorded ECG strips. These ECG strip artifacts in clinical practice must be recognized by the physician [1].

Appropriate ECG interpretation will improve patient care at ED and early referral to tertiary health center. However, studies from various countries have revealed deficiencies in ECG interpretation among medical students 
and physicians [3]. Incorrect interpretation of ECG findings can result in inappropriate management decisions with the adverse and sometimes fatal patient outcome $[1,4]$.

In Ethiopia, there have been no protocol in medical schools which guarantees competency in ECG interpretation. Therefore, this survey aimed to assess the fundamental skills in the interpretation of ECG among Ethiopian graduating medical students and analyzed whether the skills are developed during the process of medical education. This survey also evaluated the effectiveness of the teaching program on knowledge and practice regarding interpretation of the electrocardiogram (ECG) among medical interns of Addis Ababa University (AAU) and Haramaya University (HU), Ethiopia.

The objective of this survey was to assess competency and associated risk factors in ECG interpretation among graduating medical students or medical interns in AAU and HU.

\section{Methodology}

2.1. Study Area and Study Population. The survey was conducted at AAU, College of health science and HU, college of health and medical science. AAU school of medicine had emergency medicine rotation as curriculum in 4 th year. HU School of medicine did not have emergency medicine rotation as curriculum. There was no emergency medicine physician in $\mathrm{HU}$. The study population was AAU and HU 2018 graduating class medical students, who agreed to answer the questionnaire. Two-hundred ten medical interns were included to the survey based on a single population formula. On-hundred five medical interns were selected based on simple the random technique from each university.

2.2. Study Design and Period. A cross-sectional survey was employed. The survey was conducted from October 01, 2018, to October 30, 2018.

2.3. Survey Tools. ECGs were selected from the ECG textbooks and ECG web blogs. After the ECG strip selection, the previous questionnaire was modified and included in the final questionnaire. Pretested and reviewed structured selfadministered questionnaires were distributed to the survey participants. The examination consisted of basic information of GMSs and ECGs, including a specific focus on basic, arrest ECG rhythms, and common emergency ECG abnormalities strips. The ECG examination was administered to GMSs.

2.4. Data Analysis. The collected data were coded and entered to SPSS 25. Categorical variables were described as counts and percentages. Continuous variables were described as means, standard deviations, and standard errors. The Mann-Whitney $U$ test was used to compare the mean score of groups for their correct answers. The chi-squared test was used to compare differences in ECG interpretation skills, with significance level set at $P<0.05$.
Binary logistic regression was implemented to assess which factors significantly influence competency in ECG interpretation. The outcome variable was the correct answer to at least 14 questions, which is consistent with competency greater than $80 \%$; this is a commonly used threshold for a good grade on exams [3].

\subsection{Inclusion and Exclusion Criteria}

2.5.1. Inclusion Criteria. All medical students graduating in 2018 from HU and AAU, who practiced for more than 9 months, were included.

2.5.2. Exclusion Criteria. The exclusion criteria are as follows:

(A) Who are not willing or unable to participate for different reasons

(B) On attachment rotation less than 9 months because of possible lack of enough experience and awareness on EM training

2.6. Ethical Consideration. An official formal letter was written from Addis Ababa University College of health sciences, Department of Emergency and Critical Care Medicine to get permission to conduct this survey. Involvement of the participants in the survey was on a voluntary basis after getting oral consent. Confidentiality of information was maintained by removing the student's name.

\section{Results}

3.1. Characteristics of Survey Participant. In this survey, 202 graduating medical students from both HU (102 GMSs) and AAU (100 GMSs) medical schools participated and completed the self-administered questionnaires. In this survey, the nonresponse rate was $3.8 \%$. Characteristics of the survey participants are displayed in Table 1. Only eighty-two (40.6\%) of GMSs from the participant had received ECG training from medical staff other than formal medical school teaching, and $64.6 \%$ of GMSs of them were from HU.

One-hundred nineteen $(58.9 \%)$ of the participants had tried to teach themselves ECG interpretation. Of these, $33.6 \%$ use Internet, $30.3 \%$ use textbooks, and $21 \%$ of GMSs use videos as self-teaching methods.

3.2. Competency in ECG Interpretation. The percentage of accurate answers given for each of the 17 ECGs for both AAU and HU GMSs is presented in Table 2. AAU GMSs obtained a significantly higher mean score of answer 8.97 $(\mathrm{SE}=0.41)$ when compared with HU GMSs $3.33(\mathrm{SE}=0.352)$ on the 17 ECG strips. In this survey, the overall average of ECG interpretation was $35.9 \%$ (95\% CI, 32.06-39.8\%). The AAU group gained an overall average of $52.65 \%$ (95\% CI 47.88-57.41\%), while the HU group had $19.41 \%$ (95\% CI $15.47-23.71 \%)$ with $P<0.001$. Overall, in this survey, the competency in ECG interpretation was $20.8 \%$ (score $\geq 80 \%$ ). 
TABLE 1: Characteristics of the survey participant.

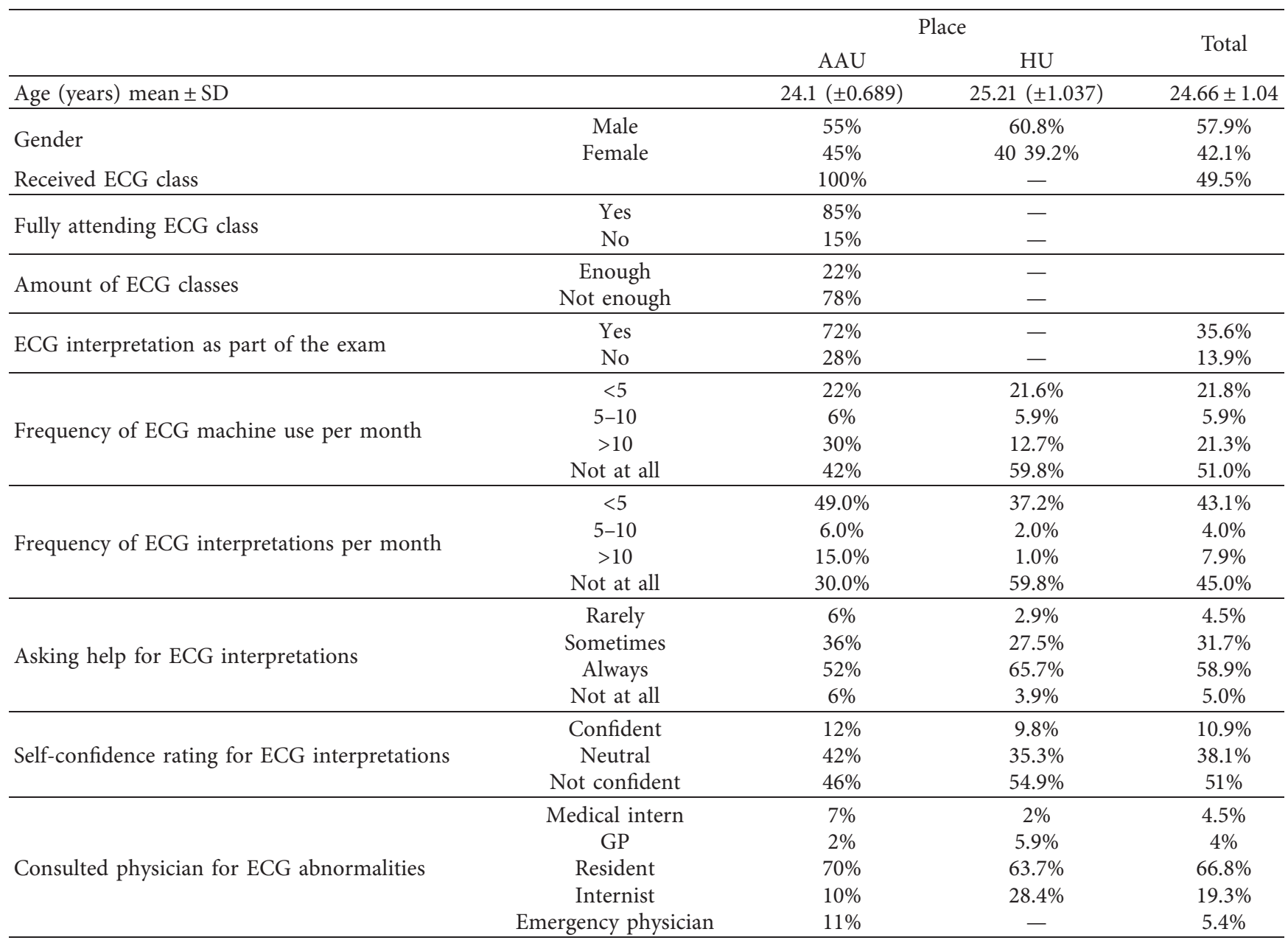

TABle 2: Correct answer for each ECG abnormalities.

\begin{tabular}{|c|c|c|c|c|}
\hline \multirow{2}{*}{ ECG finding } & \multicolumn{2}{|c|}{ Place } & \multirow{2}{*}{ Total (\%) } & \multirow{2}{*}{ Pearson chi-squared test } \\
\hline & AAU (\%) & HU (\%) & & \\
\hline Heart rate & 85.0 & 47.1 & 65.8 & $<0.001$ \\
\hline Rhythm & 91.0 & 62.7 & 76.7 & $<0.001$ \\
\hline Axis & 46.0 & 38.2 & 42.1 & 0.165 \\
\hline Asystole & 73.0 & 19.6 & 43.1 & $<0.001$ \\
\hline Ventricular tachycardia & 72.0 & 10.8 & 41.1 & $<0.001$ \\
\hline Ventricular fibrillation & 70.0 & 12.7 & 41.1 & $<0.001$ \\
\hline PEA & 5.0 & - & 2.5 & 0.028 \\
\hline Atrial fibrillation & 56.0 & 22.5 & 39.1 & $<0.001$ \\
\hline STEMI (anteroseptal) & 71.0 & 14.7 & 42.6 & $<0.001$ \\
\hline STEMI (inferior) & 41.0 & 12.7 & 26.7 & $<0.001$ \\
\hline Hyperkalemia & 45.0 & 21.6 & 33.2 & $<0.001$ \\
\hline First degree AV block & 56.0 & 18.6 & 37.1 & $<0.001$ \\
\hline Second degree AV block & 45.0 & 5.9 & 25.2 & $<0.001$ \\
\hline Third degree AV block & 38.0 & 10.8 & 24.3 & $<0.001$ \\
\hline LBBB & 42.0 & 8.8 & 26.7 & $<0.001$ \\
\hline LVH & 21.0 & 12.7 & 16.8 & 0.044 \\
\hline Pericarditis & 50.0 & 16.7 & 33.2 & $<0.001$ \\
\hline
\end{tabular}

PEA: pulseless electrical activity; STEMI: ST segment elevation myocardial infraction; AV: atrioventricular; LBBB: left bundle branch block; LVH: left ventricular hypertrophy. 
TABLE 3: Factors associated with competency of ECG interpretation.

\begin{tabular}{|c|c|c|c|c|c|}
\hline \multirow[t]{2}{*}{ Factors } & & \multicolumn{2}{|c|}{$\begin{array}{c}\text { ECG } \\
\text { Interpretation }\end{array}$} & \multirow[t]{2}{*}{ COR $(95 \%$ CI $)$} & \multirow[t]{2}{*}{ AOR (95\% CI) } \\
\hline & & $<8 \%$ & $\geq 80 \%$ & & \\
\hline \multirow{2}{*}{ Gender } & Male & 77.8 & 2.2 & $1.232(0.614-2.474)$ & \\
\hline & Female & 81.2 & 18.8 & 1 & \\
\hline \multirow{2}{*}{ ECG class } & Yes & 64.0 & 36.0 & $9.000(3.586-22.591)^{*}$ & $9.078(2.556-32.249)^{*}$ \\
\hline & No & 94.1 & 5.9 & 1 & 1 \\
\hline \multirow{2}{*}{ Place } & AAU & 64 & 36.0 & $9.000(3.586-22.591)^{*}$ & $9.078(2.556-32.249)^{*}$ \\
\hline & $\mathrm{HU}$ & 94.1 & 5.9 & 1 & 1 \\
\hline \multirow{2}{*}{ Attending all classes } & Yes & 60.0 & 40.0 & $3.923(0.826-18.634)$ & \\
\hline & No & 86.7 & 13.3 & 1 & \\
\hline \multirow{2}{*}{ Undergraduate emergency medicine rotations } & Yes & 64.0 & 36.0 & $9.000(3.586-22.591)^{*}$ & $*$ \\
\hline & No & 94.1 & 5.9 & 1 & 1 \\
\hline \multirow{2}{*}{ ECG interpretation as part of exam } & Yes & 61.1 & 38.9 & $5.273(2.543-10.935)^{*}$ & $1.031(0.363-2.932)$ \\
\hline & No & 81.7 & 18.3 & 1 & 1 \\
\hline \multirow{2}{*}{ ECG training } & Yes & 77.0 & 23.0 & $1.437(0.725-2.848)$ & \\
\hline & No & 82.0 & 18.0 & 1 & \\
\hline \multirow{3}{*}{ Confidence level } & Confident & 72.7 & 27.3 & $3.875(1.213-12.376)^{*}$ & $3.983(1.053-15.064)^{*}$ \\
\hline & Neutral & 65.4 & 34.6 & $5.471(2.39-12.522)^{*}$ & $6.057(2.362-15.533)^{*}$ \\
\hline & Not confident & 91.2 & 8.8 & 1 & 1 \\
\hline \multirow{4}{*}{ Frequency of ECG machine use per month } & $<5$ & 81.8 & 18.2 & $1.065(0.471-2.411)$ & \\
\hline & $5-10$ & 63.6 & 36.4 & $2.353(0.635-8.715)$ & \\
\hline & $>10$ & 67.9 & 32.1 & $2.229(0.862-5.765)$ & \\
\hline & Not at all & 82.5 & 17.5 & 1 & \\
\hline \multirow{4}{*}{ Frequency of ECG interpretation per month } & $<5$ & 74.7 & 25.3 & $1.924(0.870-4.256)$ & $0.901(0.349-2.326)$ \\
\hline & $5-10$ & 62.5 & 37.5 & $3.950(0.834-18.701)$ & $1.217(0.209-7.084)$ \\
\hline & $>10$ & 68.8 & 31.2 & $4.788(1.602-14.306)^{*}$ & $1.075(0.266-4.348)$ \\
\hline & Not at all & 86.8 & 13.2 & 1 & \\
\hline \multirow{4}{*}{ Ask for help for interpretation of ECG } & Rarely & 66.7 & 33.3 & $2.000(0.250-15.991)$ & \\
\hline & Sometimes & 82.8 & 17.2 & $0.830(0.155-4.455)$ & \\
\hline & Always & 78.2 & 21.8 & $1.118(0.224-5.559)$ & \\
\hline & Not at all & 80.0 & 20.0 & 1 & \\
\hline \multirow{2}{*}{ Self-learning methods } & Yes & 77.0 & 23.0 & $1.364(0.680-2.375)$ & \\
\hline & No & 83.0 & 18.0 & 1 & \\
\hline
\end{tabular}

COR: reported crude odds ratios; AOR: adjusted odds ratio $P$ : $P$ value of test statistic; CI: confidence interval; * statistically significant variable.

The competency in ECG interpretations of GMSs of AAU and $\mathrm{HU}$ who scored $\geq 80 \%$ was $36.0 \%$ and $5.9 \%$, respectively.

3.2.1. Basic ECG Findings. In this survey, 61.3\% (95\% CI $56.3-66.3 \%)$ of GMSs was able to correctly interpret the primary ECG parameters, such as heart rate, heart rhythm, and an electrical axis of the heart (Table 2). Overall, only $33.7 \%$ of GMSs was competent (>80\%) for basic ECG interpretation. Thus, three basic ECG interpretation mean scores for correct answer were 1.84; $\mathrm{SE}=0.079$. AAU GMSs had higher mean correct answer than HU GMSs (2.23; $\mathrm{SE}=0.079$ vs. $1.46 ; \mathrm{SE}=0.122$ ). The difference was statistically significant with those of the Mann-Whitney $U$ test $(U=1.84, P<0.001)$.

3.2.2. Arrest Rhythm ECG Abnormalities. The competency $(\geq 80 \%)$ of ECG interpretation was assessed for arrest rhythms, like asystole, PEA, Vtac, and Vfib; and only 3 $(1.5 \%)$ of GMSs were competent, and it is presented in Table 2. There was no GMSs from HU who answered more than $80 \%$. In this survey, the average score of arrest rhythm was $32.75 \%$ (95\% CI 28.25-37.25). AAU GMSs had higher mean correct answer than HU GMSs $(2.21 ; S E=0.016$ vs. $0.43 ; S E=0.081)$. The difference was statistically significant with that of the Mann-Whitney $U$ test $(U=1.31, P<0.001)$.

3.2.3. Common Life-Threatening Emergency ECG Abnormalities. Competency of ECG abnormalities for the list 10 ECG strips was assessed, i.e., atrial fibrillation, anterioseptal and inferior ST segment elevation myocardial infraction (STEMI), hyperkalemia, first-degree AV block, second-degree AV block, third-degree AV block, LBBB, $\mathrm{LVH}$, and pericarditis (Table 2).

In this survey, $30.4 \%$ (95\% CI 26.0-34.9\%) of GMSs were able to correctly interpret common life-threatening ECG abnormalities. Overall, only $19.3 \%$ of GMSs were competent for interpretation of common life-threatening ECG abnormalities.

3.3. Factors Affecting Competency in ECG Interpretation. In the univariable analysis, ECG class, undergraduate emergency medicine rotations, ECG interpretation as part of the exam, university of undergraduate survey, confidence level, and frequency of ECG interpretation per month were 
associated with the competency of ECG interpretation. In multivariable analysis, place of the university, ECG class, undergraduate emergency medicine rotations, and confidence level were found to have an association with competence in ECG interpretation (Table 3). Since GMSs from AAU have had ECG class and emergency medicine rotation, they were 9.078 times higher in ECG interpretation than HU GMSs $(\mathrm{AOR}=9.078(2.556-32.249))$.

\section{Discussion}

Interpretation of ECG abnormalities can be difficult. But, the ability to interpret ECG abnormalities remains a core clinical competency for GMSs and as a physician. Undergraduates do not consistently receive teaching on ECG interpretation, and this can impact confidence $[4,10,11]$. This survey also showed only GMSs from AAU have taken the ECG class.

The overall average of ECG interpretation was 35.9\% (95\% confidence interval (CI) 32.06-39.8\%). Overall, in this survey, the competency in ECG interpretation was $20.8 \%$ (score $\geq 80 \%$ ). The competency in ECG interpretations of GMSs of AAU and HU, who scored $\geq 80 \%$ were $36.0 \%$ and $5.9 \%$, respectively. Likewise, Jablonover et al. found $37 \%$ accuracy in ECG interpretation among 231 GMSs [12].

In contrast to Kopeć et al. where most students of clinical years $(86 \%)$, this survey showed lower (only two third of GMSs) ability of correctly interpret the primary ECG parameters. This survey revealed that the accuracy of ECG interpretation for arrest rhythm was lower than that in the survey done by Kopeć et al. [3]. This difference could be explained by only half of group participants took ECG class and undergraduate emergency medicine rotation.

Similar to this survey, the studies of Mahler et al. described the importance of formal ECG class which achieved a higher score than self-directed survey [13]. Against to this survey, a survey by Kopeć et al. revealed that competency in ECG interpretation was higher in students who reported ECG self-learning methods. This difference is the number of GMSs using self-learning methods was small. Likewise, McAloon et al. studied that there was a direct correlation between the confidence of GMSs and their competency to accurately identify abnormal ECG tracings.

Competency of ECG interpretation of GMSs who took undergraduate emergency medicine rotation and undergraduate ECG class was nine times more than GMSs who did not take emergency medicine rotation. But, there was no association with attending all classes, so assessment methods or teaching approaches should be assessed and changed. Similarly, a study performed at Worcester Royal Hospital on the undergraduate and postgraduate clinical training has demonstrated ECGs are interpreted suboptimal, and competency of GMSs who attended formal teaching program was significantly higher than GMSs who use self-learning methods [10].

There are several strengths to this survey. The respondents were enrolled from both medical schools, who have undergraduate emergency medicine curriculum (AAU) and who did not include HU. The simple randomized method was used to avoid bias. This survey specified the areas of life- threatening and common emergency ECG abnormalities interpretation skills that need to be improved. It clearly revealed the factors of competency of ECG interpretation. Finally, this survey reported the impact of undergraduate emergency medicine rotation as a curriculum and current ECG education in medical schools on competency in ECG interpretation.

The limitation of this survey is that ECG interpretation skill of GMSs was not assessed directly on ECG strips on the bedside. There was no nationalized curriculum in Ethiopia which assesses competency in ECG interpretation for GMSs.

\section{Conclusions}

This survey showed GMSs demonstrated low competency in ECG interpretations. Competency of ECG interpretations was significantly improved by undergraduate emergency medicine rotation and undergraduate ECG class. Unfortunately, attending all classes was not associated with competency of the ECG interpretation skill. Moreover, most students reported that the number of ECG classes during medical education was insufficient. This shows different teaching models should be applied for ECG interpretations.

\section{Recommendation}

Based on this survey finding, undergraduate emergency medicine rotation and formal ECG class should be included in a curriculum to all medical schools in Ethiopia, as this improves the accuracy of diagnosis and patient outcome. Amount of ECG class should be extended, and teaching methods should be revised and changed.

Further large study should be done on the assessment of competency of ECG interpretations among GMSs.

\section{Data Availability}

The data used to support the findings of this study are available from the corresponding author upon request.

\section{Conflicts of Interest}

The authors declare no conflicts of interest.

\section{Acknowledgments}

The authors thank the Addis Ababa University for research funding.

\section{References}

[1] A. H. Kadish, A. E. Buxton, H. L. Kennedy et al., "ACC/AHA clinical competence statement on electrocardiography and ambulatory electrocardiography: a report of the ACC/AHA/ ACP-ASIM task force on clinical competence (ACC/AHA committee to develop a clinical competence statement on electrocardiography and ambulatory electrocardiography) endorsed by the international society for holter and noninvasive electrocardiology," Journal of the American College of Cardiology, vol. 38, no. 7, pp. 2091-2100, 2001. 
[2] J. De Jager, L. Wallis, and D. Maritz, "ECG interpretation skills of South African emergency medicine residents," International Journal of Emergency Medicine, vol. 3, no. 4, pp. 309314, 2010.

[3] G. Kopeć, W. Magoń, M. Hołda, and P. Podolec, "Competency in ECG interpretation among medical students," Medical Science Monitor: International Medical Journal of Experimental and Clinical Research, vol. 21, p. 3386, 2015.

[4] M. S. A. Jensen, J. L. Thomsen, S. E. Jensen, T. Lauritzen, and M. Engberg, "Electrocardiogram interpretation in general practice," Family Practice, vol. 22, no. 1, pp. 109-113, 2004.

[5] D. C. Macallan, J. A. Bell, M. Braddick, K. Endersby, and J. Rizzo-Naudi, "The electrocardiogram in general practice: its use and its interpretation," Journal of the Royal Society of Medicine, vol. 83, no. 9, pp. 559-562, 1990.

[6] M. T. Tolla, O. F. Norheim, S. T. Memirie et al., "Prevention and treatment of cardiovascular disease in Ethiopia: a costeffectiveness analysis," Cost Effectiveness and Resource Allocation, vol. 14, no. 1, p. 10, 2016.

[7] P. Santos, P. Pessanha, M. Viana et al., "Accuracy of general practitioners' readings of ECG in primary care," Open Medicine, vol. 9, no. 3, pp. 431-436, 2014.

[8] M. Y. Yiadom, C. W. Baugh, C. M McWade et al., "Performance of emergency department screening criteria for an early ECG to identify ST-segment elevation myocardial infarction," Journal of the American Heart Association, vol. 6, no. 3, 2017.

[9] D. Eslava, S. Dhillon, J. Berger, P. Homel, and S. Bergmann, "Interpretation of electrocardiograms by first-year residents: the need for change," Journal of Electrocardiology, vol. 42, no. 6, pp. 693-697, 2009.

[10] C. McAloon, H. Leach, S. Gill, A. Aluwalia, and J. Trevelyan, "Improving ECG competence in medical trainees in a UK district general hospital," Cardiology Research, vol. 5, no. 2, p. 51, 2014.

[11] B. Little, I. Mainie, K. J. Ho, and L. Scott, "Electrocardiogram and rhythm strip interpretation by final year medical students," The Ulster Medical Journal, vol. 70, no. 2, pp. 108-110, 2001.

[12] R. S. Jablonover, E. Lundberg, Y. Zhang, and A. StagnaroGreen, "Competency in electrocardiogram interpretation among graduating medical students," Teaching and Learning in Medicine, vol. 26, no. 3, pp. 279-284, 2014.

[13] S. A. Mahler, C. J. Wolcott, T. K. Swoboda, H. Wang, and T. C. Arnold, "Techniques for teaching electrocardiogram interpretation: self-directed learning is less effective than a workshop or lecture," Medical Education, vol. 45, no. 4, pp. 347-353, 2011. 